Case Report

\title{
Personalizing Dual-Target Cortical Stimulation with Bayesian Parameter Optimization Successfully Treats Central Post-Stroke Pain: A Case Report
}

\author{
Evan M. Dastin-van Rijn ${ }^{1}\left(\mathbb{D}\right.$, Seth D. König 2,3 ${ }^{(D)}$, Danielle Carlson ${ }^{2,3}$, Vasudha Goel ${ }^{4}$, Andrew Grande ${ }^{3}$ (D), \\ Donald R. Nixdorf ${ }^{5,6}$, Sarah Benish ${ }^{7}$, Alik S. Widge ${ }^{2}$ D, Ziad Nahas ${ }^{2}$, Michael C. Park ${ }^{3,7}$, Tay I. Netoff ${ }^{1}$, \\ Alexander B. Herman ${ }^{2,+}$ and David P. Darrow ${ }^{3, *,+} \mathbb{D}$
}

1 Department of Biomedical Engineering, University of Minnesota, Minneapolis, MN 55455, USA; dasti006@umn.edu (E.M.D.-v.R.); tnetoff@umn.edu (T.I.N.)

2 Department of Psychiatry and Behavioral Sciences, University of Minnesota Medical Center, Minneapolis, MN 55454, USA; koeni117@umn.edu (S.D.K.); carl1492@umn.edu (D.C.); awidge@umn.edu (A.S.W.); znahas@umn.edu (Z.N.); herma686@umn.edu (A.B.H.)

3 Department of Neurosurgery, University of Minnesota Medical Center, Minneapolis, MN 55455, USA; grande@umn.edu (A.G.); mcpark@umn.edu (M.C.P.)

4 Department of Anesthesiology, University of Minnesota Medical Center, Minneapolis, MN 55455, USA; vgoel@umn.edu

5 Department of Diagnostic \& Biological Sciences, School of Dentistry, Minneapolis, MN 55455, USA; nixdorf@umn.edu

check for updates

Citation: Dastin-van Rijn, E.M.; König, S.D.; Carlson, D.; Goel, V.; Grande, A.; Nixdorf, D.R.; Benish, S.; Widge, A.S.; Nahas, Z.; Park, M.C.; et al. Personalizing Dual-Target Cortical Stimulation with Bayesian Parameter Optimization Successfully Treats Central Post-Stroke Pain: A Case Report. Brain Sci. 2022, 12, 25. https://doi.org/10.3390/

brainsci12010025

Academic Editor: Shapour Jaberzadeh

Received: 1 December 2021

Accepted: 22 December 2021

Published: 26 December 2021

Publisher's Note: MDPI stays neutral with regard to jurisdictional claims in published maps and institutional affiliations.

Copyright: (C) 2021 by the authors. Licensee MDPI, Basel, Switzerland. This article is an open access article distributed under the terms and conditions of the Creative Commons Attribution (CC BY) license (https:// creativecommons.org/licenses/by/ $4.0 /)$.
6 Department of Radiology, Medical School, Minneapolis, MN 55455, USA

7 Department of Neurology, University of Minnesota Medical Center, Minneapolis, MN 55455, USA; smbenish@umn.edu

* Correspondence: darro015@umn.edu; Tel.: +1-612-624-6666

+ These authors contributed equally to this work.

\begin{abstract}
Central pain disorders, such as central post-stroke pain, remain clinically challenging to treat, despite many decades of pharmacological advances and the evolution of neuromodulation. For treatment refractory cases, previous studies have highlighted some benefits of cortical stimulation. Recent advances in new targets for pain and the optimization of neuromodulation encouraged our group to develop a dual cortical target approach paired with Bayesian optimization to provide a personalized treatment. Here, we present a case report of a woman who developed left-sided facial pain after multiple thalamic strokes. All previous pharmacologic and interventional treatments failed to mitigate the pain, leaving her incapacitated due to pain and medication side effects. She subsequently underwent a single burr hole for placement of motor cortex (M1) and dorsolateral prefrontal cortex (dlPFC) paddles for stimulation with externalization. By using Bayesian optimization to find optimal stimulation parameters and stimulation sites, we were able to reduce pain from an 8.5/10 to a 0/10 during a 5-day inpatient stay, with pain staying at or below a 2/10 one-month post-procedure. We found optimal treatment to be simultaneous stimulation of M1 and dIPFC without any evidence of seizure induction. In addition, we found no worsening in cognitive performance during a working memory task with dlPFC stimulation. This personalized approach using Bayesian optimization may provide a new foundation for treating central pain and other functional disorders through systematic evaluation of stimulation parameters.
\end{abstract}

Keywords: cortical stimulation; stroke; chronic pain; electrophysiology; optimization

\section{Introduction/Background}

Central post-stroke pain (CPSP), also known as Dejerine-Roussy syndrome or thalamic pain syndrome, is a neuropathic pain syndrome that develops after a thalamic stroke. It is characterized by a period of numbness, followed by lasting and debilitating pain in the distribution of the body affected by the stroke. The pain typically fluctuates in intensity yet 
is constantly present and accompanied by altered temperature and touch sensation [1]. The pathophysiology underlying CPSP remains poorly understood, with a variety of putative mechanistic explanations proposed including changes in thalamic signaling deafferentation, hyperexcitation in damaged pathways, and imbalances in thalamo-cortical oscillatory "dialogue" [2].

CPSP remains one of the most intractable pain disorders. A variety of oral and intravenous drugs have been prescribed for treatment, including tricyclic antidepressants, anticonvulsants, and opioids, with limited response and frequent side effects [2]. Invasive neurostimulation therapies have also been attempted, including deep brain stimulation of the thalamus and periventricular gray matter and direct motor cortex stimulation. Neurostimulation efficacy rates range from $25 \%$ to $67 \%$ [2]. Unfortunately, none of these interventions has consistently proven to be long lasting, and efforts to optimize neurostimulation have not been systematic [3].

Since many of these reports were published, advancements in neuromodulation and its underlying technology have fueled innovation in novel targets, personalization, and closing the loop between electrophysiology and neurostimulation [4-6]. Recently, we have developed and tested a novel Bayesian optimization platform for objectively determining neurostimulation parameters based on patient feedback with forced binary choice [7]. This approach facilitates a methodology for continuous optimization that we hypothesized could aid with finding optimal parameters during a trial externalization for CPSP.

Multiple novel stimulation targets for central pain disorders have been proposed other than motor cortex stimulation. One area with particular promise is the dorsolateral prefrontal cortex (dIPFC) [8], with noninvasive TMS showing significant reductions in symptoms for patients with chronic neuropathic pain $[9,10]$. However, targeting the dIPFC with intracranial stimulation has not yet been tested for pain but has been successful in other indications, such as depression [11,12]. Because a second electrode can be placed through the same burrhole used for motor cortex stimulation, pairing two electrodes, one over dIPFC and the other over the M1 anatomical area, provides both convenient target selection and improved flexibility of cortical stimulation therapy for CPSP.

This case report is the first description of the use of Bayesian parameter space optimization combined with electrophysiology to treat CPSP using dual cortical stimulation in motor cortex (M1) and dlPFC. We found that combined stimulation was the most effective approach, providing more relief than either single simulation site. Simultaneous electrophysiological recordings and quantitative behavioral assessment showed no indication of epileptiform activity or cognitive impairment. We believe this approach provides a new foundation for treating central pain disorders through systematic, personalized evaluation.

\section{Case Presentation}

\subsection{Case History}

A 45-year-old woman with a medical history of multiple right sided thalamic strokes due to paradoxical emboli and an 18-month history of CPSP presented to our multidisciplinary facial pain clinic. At the time of the strokes, she experienced significant deficits, including left-sided hemiparesis and hemianesthesia. Four years later, she developed leftsided facial pain. Her facial pain began as a sensation of cold that progressed into her jaw and then up to her forehead. She described it as much worse than any headache or migraine and compared it to constant electrical shocks, rating her pain intensity as being between an 8 to 10 on a $0-10$ numerical rating scale, where 0 is no pain and 10 is as worst as imaginable. She tried multiple medications, including indomethacin (Indocin), cyclobenzaprine (Flexeril), lamotrigine (Lamictal), gabapentin (Neurontin), oxcarbazepine (Trileptal), onabotulinum toxin A (Botox), pregabalin (Lyrica), propranolol (Inderal), buspirone (Buspar), quetiapine (Seroquel), hydroxyzine, medical cannabis, erenumab (Aimovig), ubrogepant (Ubrelvy), several different opioid analgesics, and baclofen, as well as topical and subcutaneous local anesthetic. None resulted in substantial nor sustained pain relief. Her facial pain was debilitating and dominated every aspect of her life. An MRI of her brain 
demonstrated a significant focal infarct in the right thalamus (Figure 1A), and, given her history, we diagnosed her with CPSP. We offered her cortical stimulation focused on the right face region of M1 and dIPFC through a single burr hole, with inpatient externalization for trialing as a last resort therapy, with extensive discussions of the alternatives and risks.

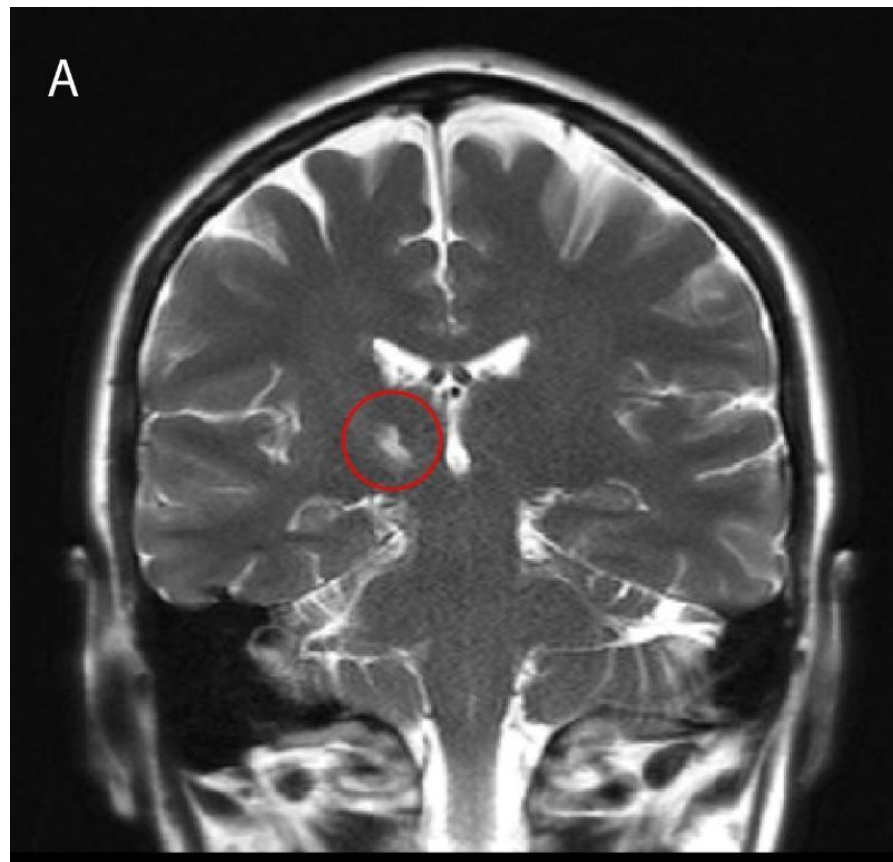

\section{B}

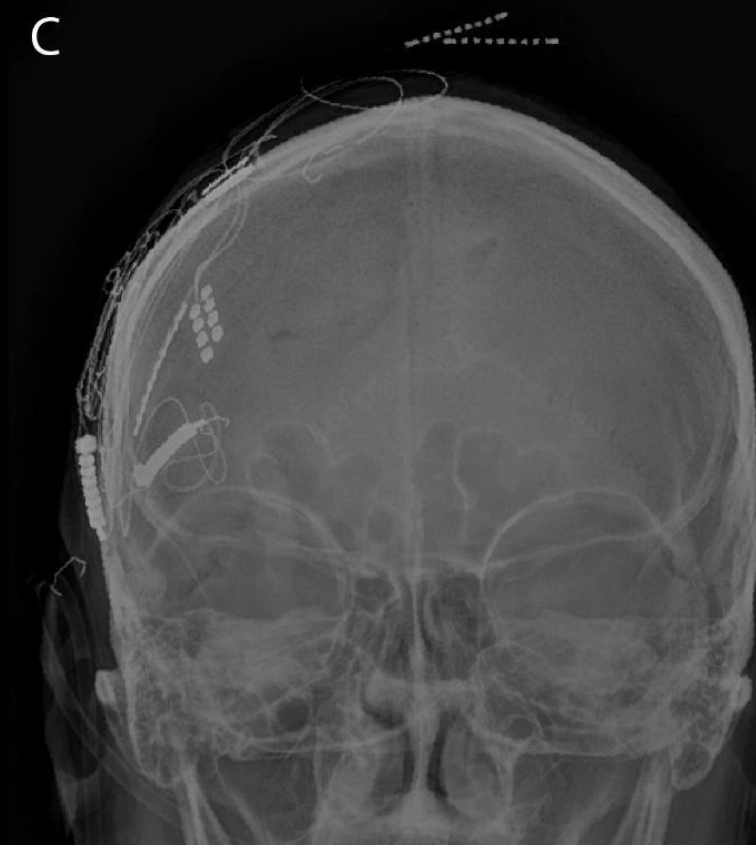

D

Figure 1. (A) T2 MR Coronal section of thalamic infarct (circled in red). (B) M1 and dlPFC electrode visualization by co-registration of the patient's MRI and CT using SPM12 toolbox in Brainstorm [13,14], green circle is the burr hole location. (C) Anteroposterior X-ray projection of electrodes. (D) Lateral X-ray projection of the two Abbott Lamitrode 44 electrodes implanted on the patient's right side. with the tunneled extensions seen superior and posteriorly. 


\subsection{Surgical Procedure: Placement of Subdural Electrodes and Externalization}

Prior to surgery, an MRI was obtained for identification of the face M1 and dlPFC targets (Figure 1A). Medtronic Stealth Neuronavigation (Medtronic, Dublin, Ireland) was used to plan the site of a burrhole superior to the M1 target along a trajectory to maximize coverage of the face portion of the motor cortex (Figure 1B). A 5-mm cutting burr was used to make this single burr hole, and the dura was sharply opened. Hemostasis was obtained, and two Lamitrode 44 electrodes (Abbott, Abbot Park, IL, USA) were placed at the target sites, which were confirmed using projections on lateral X-ray (Figure 1D). Intraoperative stimulation on the M1 electrode demonstrated evoked responses in orbicularis oculi under general anesthesia. A Guardian cranial burr hole cover (Abbott, Abbott Park, IL, USA) was used to secure the leads. Lead extensions were tunneled from a postauricular pocket to preserve the electrodes for later use, as shown in Figure 1C,D.

\subsection{Externalization Trialing}

On postoperative day 1, the broadest bipolar electrode pairs (electrodes 5-8 in M1 and 13-16 in dlPFC) were tested for a range of frequencies $(10-50 \mathrm{~Hz})$ and pulse widths (300 and $500 \mu \mathrm{s}$ ). We focused on determining safe amplitude ranges and baseline response to stimulation. Motor threshold was determined to be between $3.5-4.0 \mathrm{~mA}$ for $50 \mathrm{~Hz}$, $300 \mu$ s stimulation. She described feelings of relief and "throbbing" aligned to changes in stimulation parameters. Simultaneous electrophysiological recordings on the electrodes indicated no abnormal neural or seizure-like activity during or after stimulation.

On the second day, we systematically evaluated spatial stimulation by including four additional bipolar pairs for each paddle with varying amplitude and frequency and a constant pulse width. (Figure 2). Preference and sensation of relief varied highly with the choice of parameters, with side effects, including twitching, numbness, difficulty speaking, perceptions of itchiness, pulsing, heat, cold, and a void of feeling. During this testing, her pain varied from 5-8/10. Based on her descriptions and self-reported feeling of relief, one bipolar contact pair was chosen on each paddle (3-7 in M1 and 12-13 in dlPFC). Tonic stimulation on M1 at $2.6 \mathrm{~mA}$, frequency of $50 \mathrm{~Hz}$, and pulse width of $150 \mu \mathrm{s}$ was programmed for overnight testing. She rated her pain as a $4 / 10$ that evening.

On the third and fourth days, optimal single-site stimulation parameters (frequency, pulse width, and amplitude) were identified separately for the prior contact choices based on the result of a Bayesian parameter space optimization utilizing a probit Gaussian process to assess her preferences, similar to what we have previously described in Zhao et al. [7]. Four initial settings were chosen, from the combination of two frequencies (50 and $20 \mathrm{~Hz}$ for M1 and 26 and $50 \mathrm{~Hz}$ for dlPFC) and two pulse widths (150 and $60 \mu$ s for M1 and 150 and $300 \mu \mathrm{s}$ for dlPFC). Parameter preference was determined through a two-step process where, for each combination of frequency and pulse width, amplitude was varied, and the patient was asked to choose her preferred amplitude before comparison to previous settings, considering both pain relief and side effects. Parameter rankings were then converted to a list of all pairwise comparisons, where the win and loss was determined by the relative ranking. A probit function was then used to convert the pairwise preferences into relative values for every setting. The values for untested settings were then estimated using Gaussian Process Regression. Subsequent settings to test were selected by generating a random surface from the Gaussian Process Regression model over the range of 50-500 $\mu \mathrm{s}$ and $20-180 \mathrm{~Hz}$, with a penalizing factor for high energy parameters (powercost), and then selecting the setting with the maximum value. A total of 21 settings with 32 comparisons were made for M1 and 17 settings with 72 comparisons for dlPFC (Figure 3). She rated her pain as a 2/10 for the optimal M1 setting and as a $0 / 10$ for the optimal dIPFC setting, with no side effects observed. In both cases, the optimization resulted in good coverage of the parameter settings, but the study was limited by the duration of the clinical visit, and it cannot be shown that the model converged to a global minimum without further testing. 

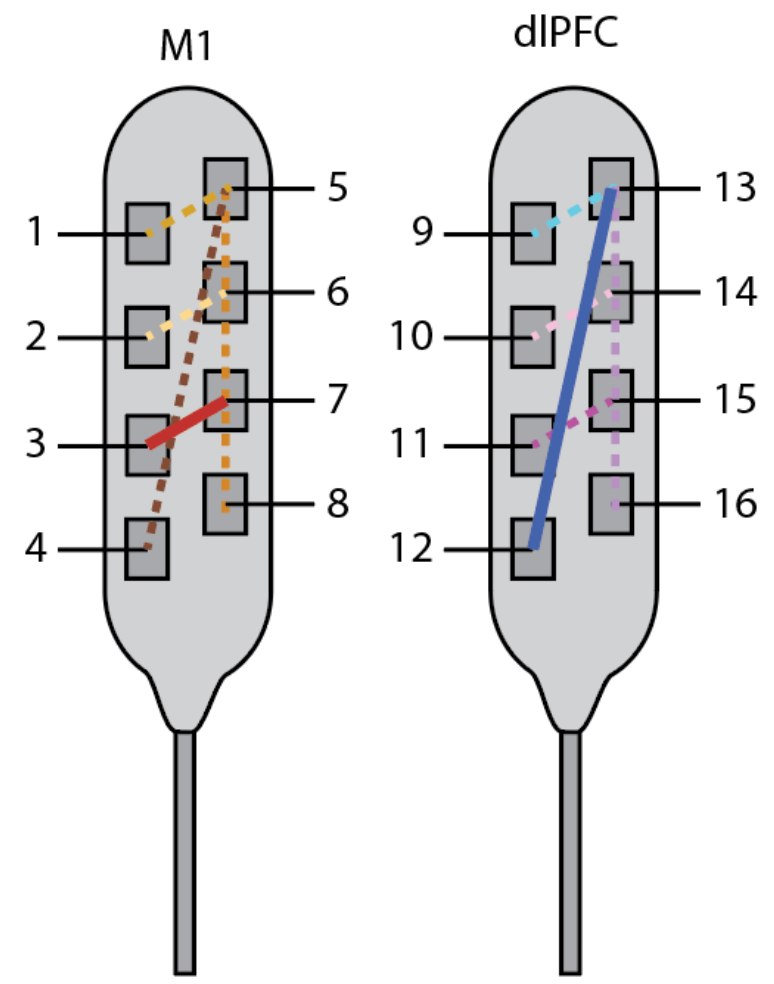

Figure 2. Day 1 and 2: parameter sweep over bipolar pairs (lines) for choice of stimulating contacts. Preferred stimulation electrodes were 3 and 7 for M1 (red solid line), and 12 and 13 for dlPFC (dark-blue solid line).

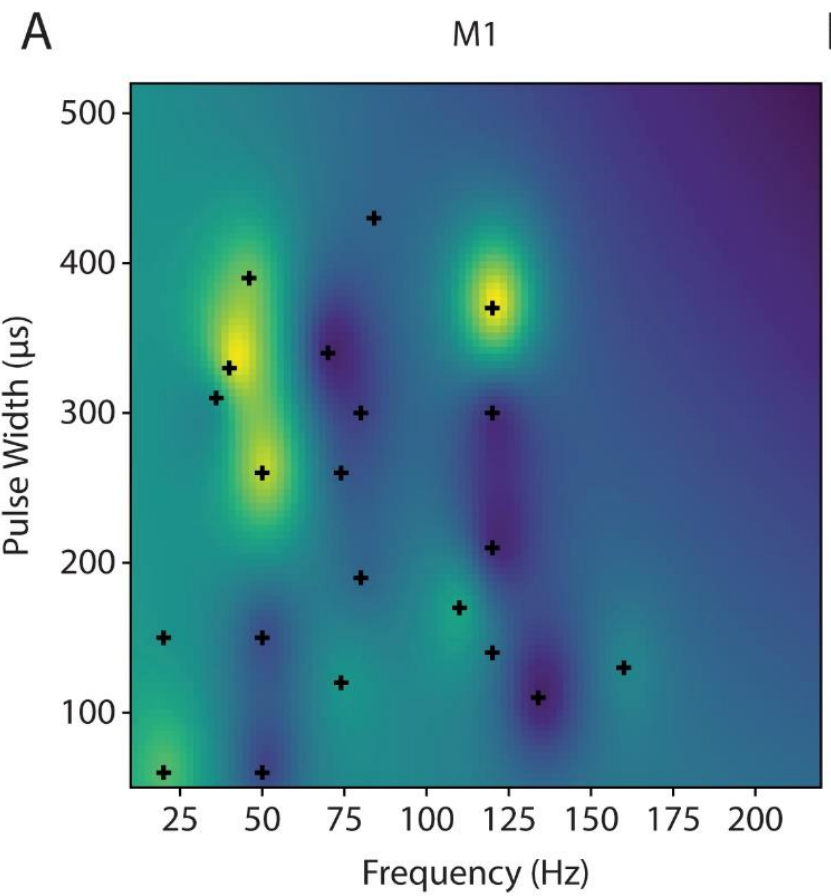

B dIPFC

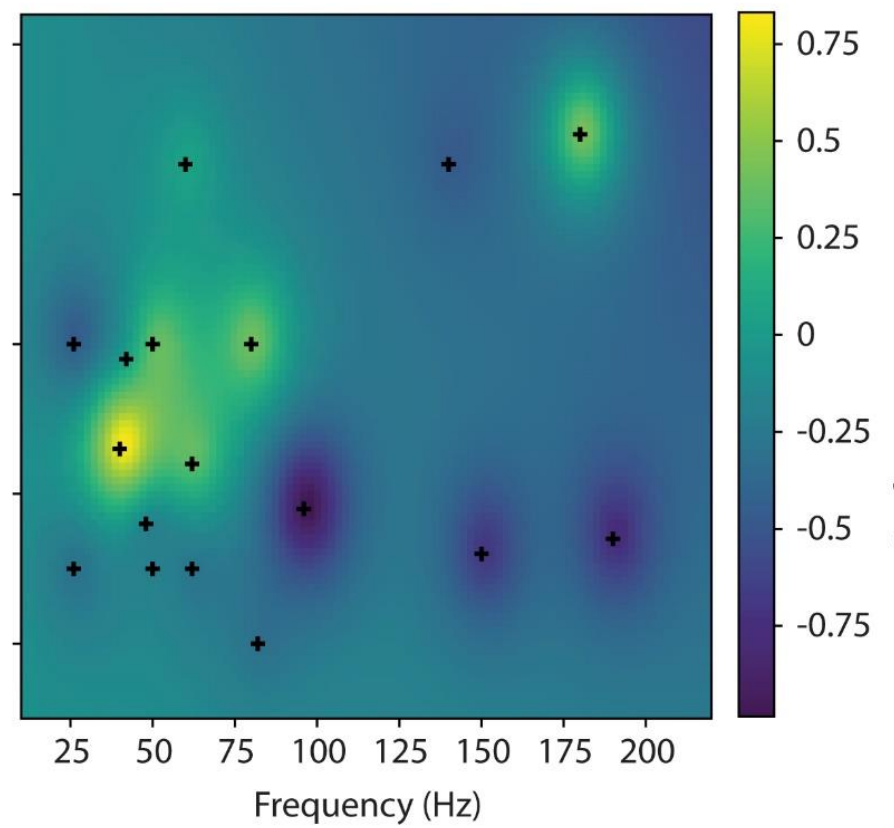

Figure 3. Frequency, pulse width, and amplitude (not shown) optimizations for single electrode combination in M1 on day 3 (A) and in dIPFC on day 4 (B). Yellower colors indicate preferred stimulation parameters, while bluer colors indicate non-preferred stimulation parameters.

Due to concerns that dlPFC stimulation might impair cognitive function, working memory was assessed at baseline and with four preferred and two non-preferred dlPFC 
stimulation settings using the N-back task [15]. The 2-back was chosen as her baseline performance was approximately $80 \%$ accurate. In total, she performed 9 blocks at baseline without stimulation and 19 blocks with stimulation. There appeared to be some variability in N-back performance metrics across specific dIPFC stimulation settings (Figure 4). However, a Wilcoxon rank sum test showed that, across all dlPFC stimulation parameters, stimulation did not significantly affect accuracy $(p=0.23)$ or reaction times $(p=0.17)$. While her performance did not change with stimulation, she sometimes reported increased difficulty during stimulation blocks, suggesting that dIPFC stimulation may modulate perceived cognitive effort.

A

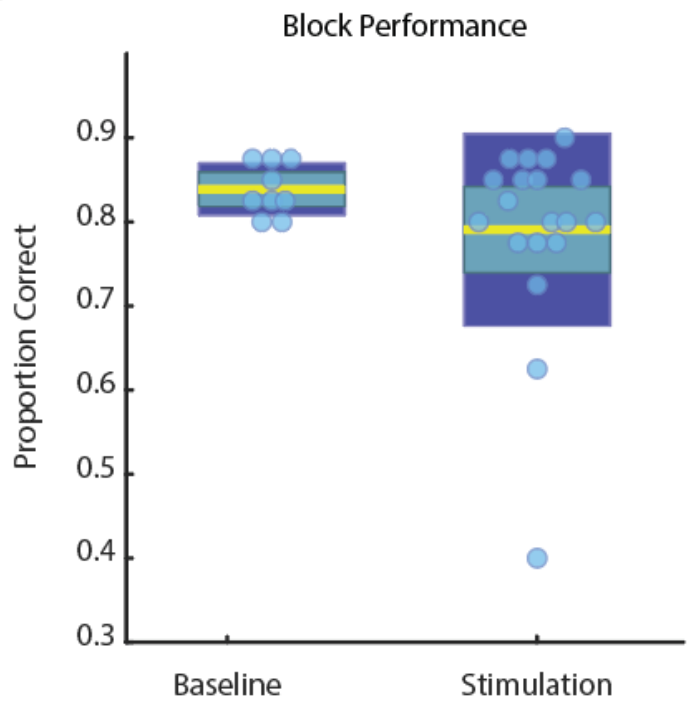

B

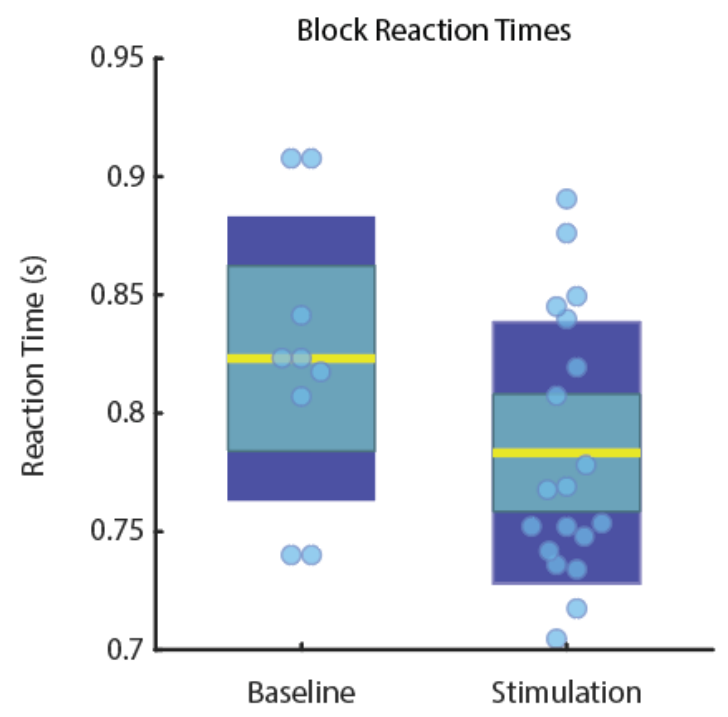

Figure 4. Performance (A) and reaction time $(\mathbf{B})$ on the 2-back was comparable for baseline $(n=9)$ and dIPFC stimulation $(n=19)$ blocks, suggesting stimulation did not significantly impair cognitive function. Data for each block are indicated by the light-blue circles, standard deviation in dark-blue, standard error in teal, and the mean in yellow.

On the fifth day, dual site M1 and dIPFC stimulation was tested in a blinded fashion against previous independent settings. Following extended testing and comparison between relieving parameter combinations, we found that the dual site stimulation reliably provided the most relief compared to either single site in isolation.

\subsection{Effects of Stimulation on Electrophysiology}

While no seizures were observed at any time during or after stimulation, we were concerned that the stimulation may trigger subclinical epileptiform electrophysiological activity. We reviewed post-stimulation cortical activity and found no significant afterdischarges or noticeable difference in activity upon visual review (Figure 5A). Simple threshold-based analysis of broadband signals also did not identify the presence of seizurelike activity. 
A
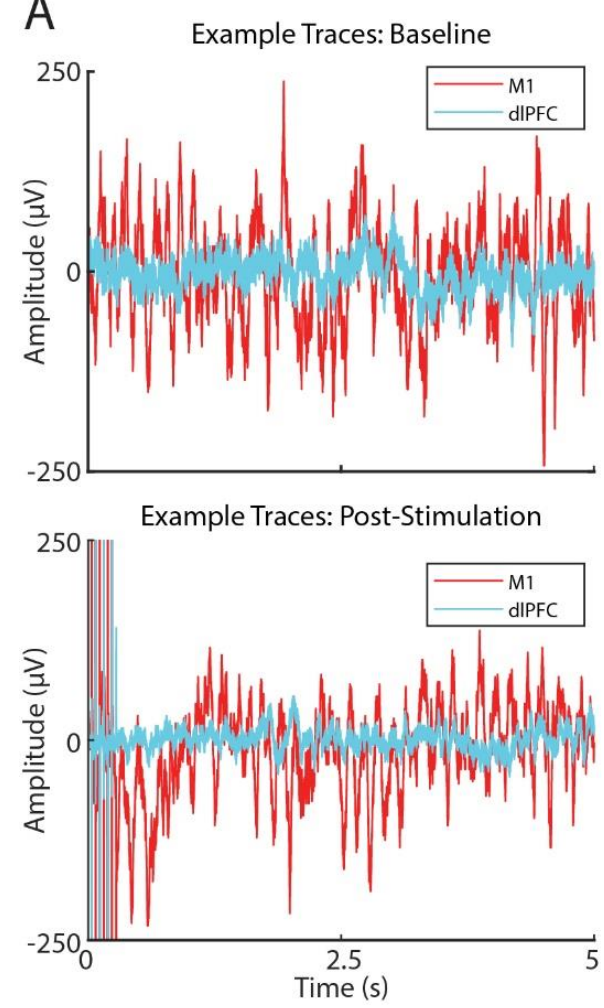

B

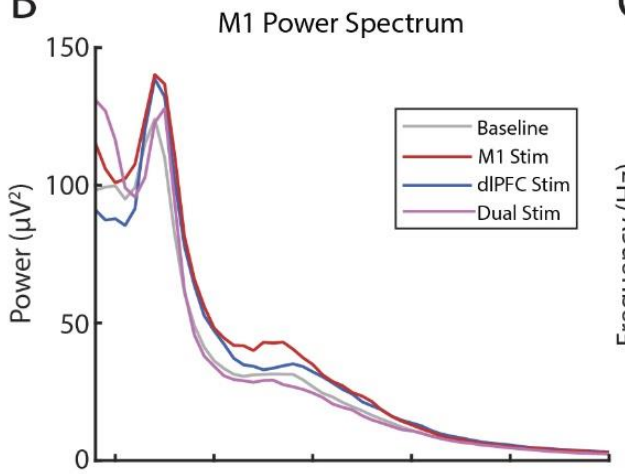

dIPFC Power Spectrum

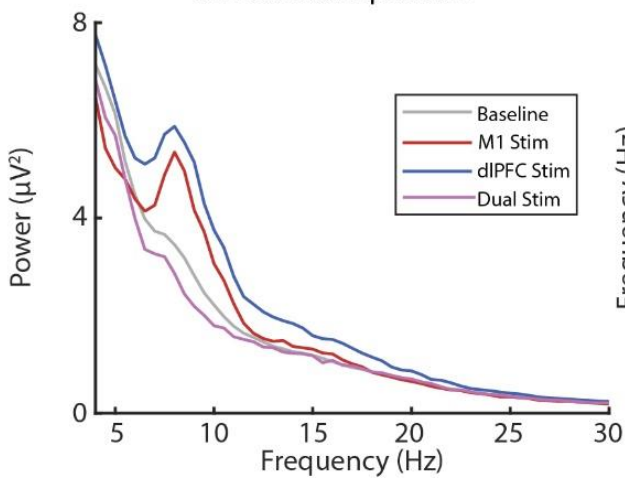

C

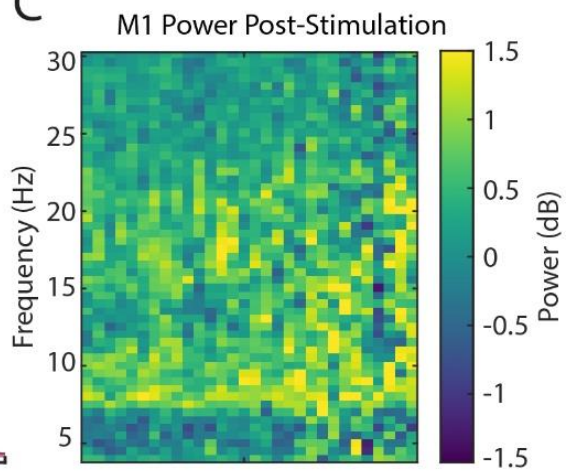

dIPFC Power Post-Stimulation

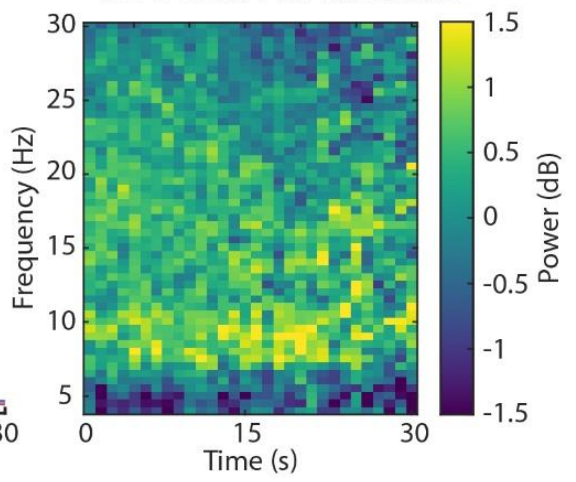

Figure 5. Effects of stimulation on M1 and dLPFC activity. (A) Voltage-time traces of M1 (red) and dlPFC activity (cyan) at baseline (top) and post-stimulation (bottom). (B) Power spectra at baseline and following M1 (maroon), dlPFC (dark blue), or dual-site stimulation (purple) in M1 (top) and dlPFC (bottom). (C) Spectrograms of M1 (top) and dIPFC (bottom) power post-stimulation normalized to the baseline period.

While no concerning changes in activity were observed, we noticed some changes in spectral power immediately following stimulation. We combined all post-stimulation recording periods to quantify stimulation-induced changes in cortical activity. Using FieldTrip to calculate power spectra [16], we built several Generalized Linear Mixed-Effects Models (GLMEs, bandPower $\sim 1$ + timeWindow + stimulationLocation + (1 I channelNumber)) to determine if bandPower in specific brain areas varied with stimulation location, and we found significant (GLME, $p<0.05)$ power changes in theta $(6-9 \mathrm{~Hz})$ and low beta (13-20 Hz) bands following stimulation (Table 1 and Figure 5B). Interestingly, individually, M1 and dIPFC stimulation tended to increase theta- and beta-band power at both sites, while dual site stimulation tended to decrease power (GLME, $p<0.05$ ). There was weak evidence that theta and beta power changed over time post-stimulation (Figure 5C, GLME, $p<0.05$ ), but effect sizes were all below $0.014 \mu \mathrm{V}^{2} \mathrm{~s}^{-1}$; moreover, both positive and negative effects were observed suggesting these effects are unreliable across recording locations and stimulation parameters. Additionally, we did not find any power effects based on specific stimulation parameters; this may be due to our focus on finding optimized stimulation parameters for pain relief rather than for repeated measures analysis. 
Table 1. GLME results showing effect of stimulation on theta- $(6-9 \mathrm{~Hz})$ and low beta-band $(13-20 \mathrm{~Hz})$ power.

\begin{tabular}{ccccc}
\hline Stim Target & Power Band & Recording Location & $\boldsymbol{p}$-Value & Effect Size $\left(\boldsymbol{\mu} \mathbf{V}^{\mathbf{2}}\right)$ \\
\hline M1 & Theta & M1 & 0 & 23.8 \\
M1 & Theta & dlPFC & 0 & 2.22 \\
M1 & Beta & M1 & $1.21 \times 10^{-12}$ & 3.30 \\
M1 & Beta & dlPFC & 0.115 & -0.0667 \\
dlPFC & Theta & M1 & 0 & 13.4 \\
dlPFC & Theta & dlPFC & 0 & 3.87 \\
dlPFC & Beta & M1 & $9.97 \times 10^{-2}$ & 0.613 \\
dlPFC & Beta & dlPFC & 0 & 0.471 \\
M1/dlPFC & Theta & M1 & $5.84 \times 10^{-3}$ & 2.69 \\
M1/dlPFC & Theta & dlPFC & 0 & -1.17 \\
M1/dlPFC & Beta & M1 & 0 & -4.89 \\
M1/dlPFC & Beta & dlPFC & $7.59 \times 10^{-8}$ & -0.170 \\
\hline
\end{tabular}

\subsection{Final Treatment Plan}

Overall, pain ratings decreased from a baseline of $8.5 / 10$ to $0 / 10$ following stimulation parameter optimization. Based on this change, she underwent permanent implantation of the cortical stimulation system. Under general anesthesia, the previous post-auricular incision was opened, and the electrode extension leads were disconnected, cut, and carefully removed to avoid contamination. New extensions were connected to both paddles and tunneled subcutaneously to an implantable pulse generator (IPG) placed over the right chest. The settings that provided maximal pain relief were programmed into the IPG, and the system was configured for remote programming to continue the forced-choice Bayesian optimization remotely using NeuroSphere ${ }^{\mathrm{TM}}$ (Abbott, Abbott Park, IL, USA). At one-month post-procedure, she reported pain levels no higher than $2 / 10$ at any time, and we began remote programming to continue to search for optimal settings over time using our Bayesian optimization.

\section{Discussion}

CPSP affects between 1 and $12 \%$ of stroke patients with higher rates for experiencing pain following strokes in the thalamus and lateral medulla. Despite the prevalence of CPSP, it remains poorly understood and difficult to treat [2].

Off-label neurostimulation therapies, such as motor cortex stimulation, are considered in treatment-resistant cases of CPSP. While the mechanisms underlying the effect of motor cortex stimulation are currently unknown, prior case series have implicated changes in cerebral blood flow and thalamic bursting [2]. Reviews of these case series estimated the 1 -year success of motor cortex stimulation to be between $45-50 \%$ (2). Despite this, we have not observed efforts to implement a systematic approach to personalize stimulation. Therefore, we implemented a forced-choice Bayesian preference optimization to be used long-term with remote programming during an inpatient externalization where feasibility was demonstrated and encouraging results found.

Noninvasive studies have shown that dIPFC function is abnormally increased in patients with chronic pain, and non-invasive stimulation of the dlPFC has been reported to be effective for alleviating such pain. Converging evidence suggests that dlPFC has a role in cognitive components of pain, acting as an interface between cognitive processing and pain regulation [8]. Considering the inconsistency of motor cortex stimulation and preliminary evidence of dlPFC's role in pain processing, we elected to target both regions with the aim of treating the patient's pain in an individualized multifaceted manner. While dlPFC plays a critical role in working memory performance, we did not observe a significant performance decrease in working memory function during various dIPFC stimulation regimes, though there was some variation in task performance with different stimulation parameters. Furthermore, some settings were perceived oddly (e.g., feeling "spacey"), 
yet were sometimes associated with increases in target or non-target performance. These results imply that dIPFC stimulation may be biasing attention towards or away from certain environmental features (e.g., targets), which may be similarly beneficial for biasing attention away from pain. While the exact mechanism by which the stimulation relieves pain is unknown, the electrophysiology suggests that stimulation produces short-term plastic changes in the recorded networks observed here as changes in theta- $(6-9 \mathrm{~Hz})$ and low betaband $(13-20 \mathrm{~Hz})$ power following stimulation. These frequency bands are known to play an important role in interareal communication, attention, and working memory [17-19]. Together with electrophysiology demonstrating no significant after discharges or seizurelike activity, these results suggest invasive stimulation of dIPFC may prove to be a promising and safe target for various aspects of chronic, intractable pain.

Our results at first appear inconsistent with the one randomized controlled trial to examine repetitive TMS to the dIPFC for CPSP, which found limited efficacy [20]. However, intracranial stimulation has a more direct effect on the cortical substrate and has been demonstrated to have efficacy in other clinical settings where repetitive TMS has failed $[11,12]$. Furthermore, this previous study did not pursue an optimization strategy, which we view as central to our outcome.

We observed that dual dIPFC and M1 stimulation efficacy was greater than M1 or dIPFC stimulation alone, suggesting a promising new approach for treating CPSP. Dual stimulation was also associated with a qualitatively different spectral power response: a decrease in theta and beta power in both regions, in contrast to the increase seen with single site stimulation. The current case demonstrates the significant heterogeneity in response dependent on the settings chosen, motivating the necessity of methods, such as Bayesian parameter space optimization, to ensure treatment is accurately tailored to a patient's condition. Additionally, newer stimulation systems have features for remote programming, providing a platform by which parameters could be continuously optimized away from the clinic via a simple video visit [21]. The ability to identify multiple, similarly effective yet largely different parameter combinations will provide patients and clinicians with more flexibility as preferences and symptoms vary, which we hypothesize may improve longterm outcomes. Exploring new targets and stimulation settings prompts us to also consider the potential side effects, such as impairments in cognition or seizure generation, for which quantitative behavioral testing and electrophysiology can provide helpful platforms.

\section{Conclusions}

We provide a single case report demonstrating the possible utility of Bayesian preference parameter space optimization and a dual site, M1 and dlPFC, stimulation protocol to treat CPSP through a single burr hole. The approach of dual-site stimulation in dIPFC and M1 may be considered for treatment of patients with CPSP who do not respond to conventional pharmaceuticals and noninvasive procedures. The combination of both sites and systematic parameter optimization are associated with significant reductions in pain without evidence of side effects or reductions in cognitive performance.

Author Contributions: Conceptualization, D.P.D., A.B.H., A.S.W. and T.I.N.; methodology, D.P.D., A.B.H., A.G., V.G., S.B., A.S.W., Z.N., M.C.P. and T.I.N.; software, T.I.N. and S.D.K.; formal analysis, S.D.K.; investigation, D.P.D., T.I.N., S.D.K. and E.M.D.-v.R.; resources, D.P.D., A.B.H. and T.I.N.; data curation, S.D.K.; writing—original draft preparation, E.M.D.-v.R., S.D.K., D.C. and D.P.D.; writingreview and editing, E.M.D.-v.R., D.P.D., S.D.K., A.B.H., D.R.N., A.S.W., D.C. and S.B.; visualization, E.M.D.-v.R., S.D.K. and D.C.; supervision, D.P.D., T.I.N.; project administration, D.P.D. All authors have read and agreed to the published version of the manuscript.

Funding: This research received no external funding.

Institutional Review Board Statement: All procedures performed in the studies in accordance with the ethical standards of the institutional and/or national research committee and with the 1964 Helsinki Declaration and its later amendments or comparable ethical standards. 
Informed Consent Statement: Written informed consent has been obtained from the patient to publish this paper.

Data Availability Statement: The data presented in this study are available on request from the corresponding author.

Conflicts of Interest: D.P.D and T.I.N hold equity in and serve as officers for a neuromodulation company (Stimsherpa).

\section{References}

1. Keszler, M.; Gude, T.; Heckert, K. Chapter 19-Pain Syndromes Associated With Cerebrovascular Accidents. In Challenging Neuropathic Pain Syndromes; Freedman, M.K., Gehret, J.A., Young, G.W., Kamen, L.B., Eds.; Elsevier: Amsterdam, The Netherlands, 2018; pp. 155-165, ISBN 978-0-323-48566-1.

2. Klit, H.; Finnerup, N.B.; Jensen, T.S. Central Post-Stroke Pain: Clinical Characteristics, Pathophysiology, and Management. Lancet Neurol. 2009, 8, 857-868. [CrossRef]

3. Fontaine, D.; Hamani, C.; Lozano, A. Efficacy and Safety of Motor Cortex Stimulation for Chronic Neuropathic Pain: Critical Review of the Literature. J. Neurosurg. 2009, 110, 251-256. [CrossRef] [PubMed]

4. $\quad$ Scangos, K.W.; Khambhati, A.N.; Daly, P.M.; Makhoul, G.S.; Sugrue, L.P.; Zamanian, H.; Liu, T.X.; Rao, V.R.; Sellers, K.K.; Dawes, H.E.; et al. Closed-Loop Neuromodulation in an Individual with Treatment-Resistant Depression. Nat. Med. 2021, 27, 1696-1700. [CrossRef] [PubMed]

5. Allawala, A.; Bijanki, K.R.; Goodman, W.; Cohn, J.F.; Viswanathan, A.; Yoshor, D.; Borton, D.A.; Pouratian, N.; Sheth, S.A. A Novel Framework for Network-Targeted Neuropsychiatric Deep Brain Stimulation. Neurosurgery 2021, 89, E116-E121. [CrossRef] [PubMed]

6. Widge, A.S.; Malone, D.A.; Dougherty, D.D. Closing the Loop on Deep Brain Stimulation for Treatment-Resistant Depression. Front. Neurosci. 2018, 12, 175. [CrossRef] [PubMed]

7. Zhao, Z.; Ahmadi, A.; Hoover, C.; Grado, L.; Peterson, N.; Wang, X.; Freeman, D.; Murray, T.; Lamperski, A.; Darrow, D.; et al. Optimization of Spinal Cord Stimulation Using Bayesian Preference Learning and Its Validation. IEEE Trans. Neural Syst. Rehabil. Eng. 2021, 29, 1987-1997. [CrossRef] [PubMed]

8. Seminowicz, D.A.; Moayedi, M. The Dorsolateral Prefrontal Cortex in Acute and Chronic Pain. J. Pain 2017, 18, 1027-1035. [CrossRef] [PubMed]

9. Sampson, S.M.; Kung, S.; McAlpine, D.E.; Sandroni, P. The Use of Slow-Frequency Prefrontal Repetitive Transcranial Magnetic Stimulation in Refractory Neuropathic Pain. J. ECT 2011, 27, 33-37. [CrossRef] [PubMed]

10. Nardone, R.; Höller, Y.; Langthaler, P.B.; Lochner, P.; Golaszewski, S.; Schwenker, K.; Brigo, F.; Trinka, E. RTMS of the Prefrontal Cortex Has Analgesic Effects on Neuropathic Pain in Subjects with Spinal Cord Injury. Spinal Cord 2017, 55, 20-25. [CrossRef]

11. Nahas, Z.; Anderson, B.S.; Borckardt, J.; Arana, A.B.; George, M.S.; Reeves, S.T.; Takacs, I. Bilateral Epidural Prefrontal Cortical Stimulation for Treatment-Resistant Depression. Biol. Psychiatry 2010, 67, 101-109. [CrossRef] [PubMed]

12. Williams, N.R.; Short, E.B.; Hopkins, T.; Bentzley, B.S.; Sahlem, G.L.; Pannu, J.; Schmidt, M.; Borckardt, J.J.; Korte, J.E.; George, M.S.; et al. Five-Year Follow-Up of Bilateral Epidural Prefrontal Cortical Stimulation for Treatment-Resistant Depression. Brain Stimul. 2016, 9, 897-904. [CrossRef] [PubMed]

13. Ashburner, J.; Friston, K.J. Unified Segmentation. NeuroImage 2005, 26, 839-851. [CrossRef] [PubMed]

14. Tadel, F.; Baillet, S.; Mosher, J.C.; Pantazis, D.; Leahy, R.M. Brainstorm: A User-Friendly Application for MEG/EEG Analysis. Comput. Intell. Neurosci. 2011, 2011, 879716. [CrossRef] [PubMed]

15. Kirchner, W.K. Age Differences in Short-Term Retention of Rapidly Changing Information. J. Exp. Psychol. 1958, 55, 352. [CrossRef] [PubMed]

16. Oostenveld, R.; Fries, P.; Maris, E.; Schoffelen, J.-M. FieldTrip: Open Source Software for Advanced Analysis of MEG, EEG, and Invasive Electrophysiological Data. Comput. Intell. Neurosci. 2011, 2011, 156869. [CrossRef] [PubMed]

17. Reinhart, R.M.G. Disruption and Rescue of Interareal Theta Phase Coupling and Adaptive Behavior. Proc. Natl. Acad. Sci. USA 2017, 114, 11542-11547. [CrossRef] [PubMed]

18. Clayton, M.S.; Yeung, N.; Cohen Kadosh, R. The Roles of Cortical Oscillations in Sustained Attention. Trends Cogn. Sci. 2015, 19, 188-195. [CrossRef] [PubMed]

19. Miller, E.K.; Lundqvist, M.; Bastos, A.M. Working Memory 2.0. Neuron 2018, 100, 463-475. [CrossRef] [PubMed]

20. de Oliveira, R.A.A.; de Andrade, D.C.; Mendonça, M.; Barros, R.; Luvisoto, T.; Myczkowski, M.L.; Marcolin, M.A.; Teixeira, M.J. Repetitive Transcranial Magnetic Stimulation of the Left Premotor/Dorsolateral Prefrontal Cortex Does Not Have Analgesic Effect on Central Poststroke Pain. J. Pain 2014, 15, 1271-1281. [CrossRef]

21. Sharma, V.D.; Safarpour, D.; Mehta, S.H.; Vanegas-Arroyave, N.; Weiss, D.; Cooney, J.W.; Mari, Z.; Fasano, A. Telemedicine and Deep Brain Stimulation-Current Practices and Recommendations. Parkinsonism Relat. Disord. 2021, 89, 199-205. [CrossRef] [PubMed] 